\title{
Anti-Single Stranded DNA IgG Measurement
}

National Cancer Institute

\section{Source}

National Cancer Institute. Anti-Single Stranded DNA IgG Measurement. NCI Thesaurus. Code C92269.

The determination of the amount of anti-single stranded DNA IgG present in a sample. 